\title{
A particle image velocimetry study of dual-rotor counter-rotating wind turbine near wake
}

Received: 13 June 2019/Accepted: 3 October 2019/Published online: 31 March 2020

(C) The Author(s) 2020

\begin{abstract}
This experimental work studied the flow characteristics in the near wake region behind dual-rotor wind turbines using two-dimensional particle image velocimetry. Two auxiliary rotors of $50 \%$ and $80 \%$ scale of the main rotor were installed upwind and operated in counter-rotating condition, which are compared to the conventional single-rotor turbine. In all the three configurations, a constant Reynolds number $9.5 \times 10^{4}$ was applied, and all the rotors operated at a fixed tip speed ratio of 3.46. The mean and phaseaveraged velocity fields were investigated together with the turbulence kinetic energy. It was found that the two auxiliary rotors do not result in a significantly different wake flow property. The configuration implementing the $50 \%$ auxiliary rotor sees a slightly better wake characteristics, in terms of weaker main rotor tip vortices and a counter-rotating swirling shear region at the mid-span behind the main rotor. The decay rates of the peak vorticity of the main rotor tip vortices and their circulation are found to follow an exponential manner.
\end{abstract}

Keywords Wind turbine $\cdot$ Dual-rotor $\cdot$ Near wake $\cdot$ PIV

\section{Introduction}

The global wind industry is expected to surpass generation of $60 \mathrm{GW}$ in 2020, and reach a total of $840 \mathrm{GW}$ by 2022 (Global Wind Energy Council 2017). These values alone highlight the significance of improvements to wind turbine efficiency. An increase as small as 5\% could result in an additional $42 \mathrm{GW}$ of clean, emissions-free wind power by the year 2020. The majority wind turbines are single rotor (SRWT) type. Current SRWTs are limited at the hands of three main factors: the inherent Betz limit, root losses and wake interactions. The Betz limit defines the theoretical maximum efficiency of a horizontal-axis single-rotor wind turbine stating that no more than $59.3 \%$ of the kinetic energy from wind may be converted into useful mechanical energy to turn the rotor. Root losses are the result of thick and thus aerodynamically poor turbine blade roots required to withstand large structural loads; it is stated that a loss in power generation of up to $5 \%$ is approximated for horizontal axial wind turbines due to increased structural integrity required at the root (Sharma and Frere 2010). Wake interactions are relevant specifically to wind farms featuring a configuration of many wind turbines as opposed to isolated use. The problem is that a resultant wake after passing through a turbine expands, superimposes and impinges upon downstream turbines negatively affecting the wake, and consequently, the downwind turbine's ability to extract energy from it. The combined effect of these three limitations sees an efficiency of approximately between $10 \%$ and $30 \%$ for conventional SRWTs.

E. O. Hollands · C. He · L. Gan ( ()

Department of Engineering, Durham University, Durham DH1 3LE, UK

E-mail: lian.gan@durham.ac.uk 
In the context of a wind farm, it is desired that the wake passing through a turbine recovers quickly so as not to inhibit the functionality of the downstream turbine and reduce the possible harmful resonance on the blades. However, there are several factors that prohibit the recovery of a wake, the most detrimental of which is the phenomenon of tip vortices. The pressure difference responsible for generating lift at an airfoil surface also causes vortices emanating from the blade tips whose pathlines are helical in nature due to the rotation of the turbine blades (Sherry et al. 2013). As the speed of the turbine blade tips is significantly higher than the incoming flow speed, the distance between the spirals of the tip vortices is very small meaning they can be approximated as a very turbulent cylindrical shear layer, separating the wake containing slow moving air and the surrounding fluid at ambient conditions (Gomez-Elviraa et al. 2005). This shear layer essentially acts as a barrier, delaying wake re-energisation via mixing with ambient air. Furthermore, it is suggested by Bartl (2011) that the swirling motion leaving the turbine rotor could excite an eigenfrequency of the blades of the downstream turbine leading to material fatigue. Consequently, a method of dissipating these vortices as soon as possible is desired.

This evident need for improvement sparked the relatively new research into other unconventional wind turbine designs, such as horizontal dual-rotor wind turbine (DRWT). DRWTs are characterised by two rotors mounted on a single tower to both capture additional energy otherwise missed by a single rotor and, more importantly, ultimately to improve the characteristics of the wake. Ozbay (2014) conducted wind tunnel experiments and compared SRWT and DRWT for both co-rotating and counter-rotating configurations. The study found that the dissipation of the vortex-induced shear layer is highly dependent on the turbulence kinetic energy (TKE) available for turbulent mixing. This need for increased TKE as an input to the wake is met by the DRWT, experimentally validated by incorporating a smaller auxiliary rotor half of the size of the main rotor (Wang et al. 2018). It found improved recovery in the wake of a DRWT compared to that of a SRWT due to the interaction and consequent dissipation of separately emanating tip vortices. The measurements also concluded that although the highest turbulence production rate was seen behind the co-rotating DRWT, it suffered the largest velocity deficit and was unable to utilise the swirling velocity induced by the upwind rotor as the counter-rotating DWRT could. The counter-rotating and co-rotating DRWT saw power enhancements of $7.2 \%$ and $1.8 \%$, respectively, providing confidence in the decision to go for the former configuration.

Herzog et al. (2010) compared both numerically and experimentally a SRWT with a counter-rotating DRWT in which both rotors were of the same diameter. In an effort to more accurately simulate the free stream operation of both turbine configurations, the blockage effects of the wind tunnel used were numerically studied as well as practical measurement of the drag coefficient. It was concluded that an increase in power output of 9\% was achieved when compared to the SRWT. Lee et al. (2012) studied the effects of design parameters on the aerodynamic performance of a counter-rotating DRWT and concluded that for optimal system performance, the secondary rotor should be about $40 \%-50 \%$ the size of the larger rotor, depending on the pitch of each rotor. This also agreed with the field testing (Jung et al. 2005). The optimal power generation was later further studied parametrically (Rosenberg et al. 2014), which however found that the auxiliary rotor placed upwind of the existing rotor should have a diameter $25 \%$ the size of the latter, combined with $2 D$ ( $D$ being the diameter of the main rotor) distance and a tip speed ratio of 6 , to yield optimal system performance.

Although these studies align closely with the intentions of the current study, their primary focus was on the power output of a standalone system, while it is important to know the wake characteristics are not wellunderstood, which however are crucial for eventual large-scale implementation involving multiple DRWT systems. This study aims to focus on the near wake behind a counter-rotating DRWT. The dependence of the helical vortex wake decay on the size of the smaller auxiliary rotor is of the special interest. The primary advantage of this configuration is twofold; the smaller rotor placed upstream aims to capture the energy loss on the root of the main turbine more economically than using the same size rotor, while the opposite rotating direction is potentially beneficial to counteract the swirl or at least accelerate the swirl decay behind the DRWT system. 


\section{Experimental set-up}

\subsection{Turbine model and its operation}

A scaled turbine tower and nacelle were manufactured for use in a wind tunnel of $0.5 \mathrm{~m}$ working section. The turbine tower is $225 \mathrm{~mm}$ long and extended vertically downwards from the ceiling, placing the nacelle at the centre of the testing section. The main rotor diameter is $180 \mathrm{~mm}$, sufficiently small so as to avoid flow interference with the wind tunnel wall. The rotors embodied a NACA 4415 aerofoil profile, being one of the most common and broadly used aerodynamic shapes for wind turbine blades, and were 3D printed by FullCure ${ }^{\circledR} 720$ using an Eden Object 500 printer. This produced fairly accurate blade shape and good surface finish, ready to be spray-painted matte black straight after curing. The largest rotor provided the base model of which the two auxiliary rotors were simply scaled versions at factors of 0.8 and 0.5 to ensure aerodynamic similarity.

Three turbine configurations were studied: a SRWT comprised of the main rotor only, a DRWT that modifies the SRWT model to include a 144-mm-diameter rotor (80\%), placed $60 \mathrm{~mm}$ (the length of the hub) upstream, and a second DRWT that utilises the smaller 90-mm-diameter rotor (50\%); see Fig. 1a. Smaller rotor size was difficult to achieve due to the 3D printer resolution as well as the material strength. DWRTs having auxiliary rotors larger than $80 \%$ of the main rotor will not be economically suitable in practice compared to two separate single-rotor wind turbines. Hence, they are not studied.

In the two DRWT configurations, the smaller auxiliary rotor was a mirror of the main and was placed upwind, aiming to capture the energy at the root part of the main rotor installed at the downstream side of
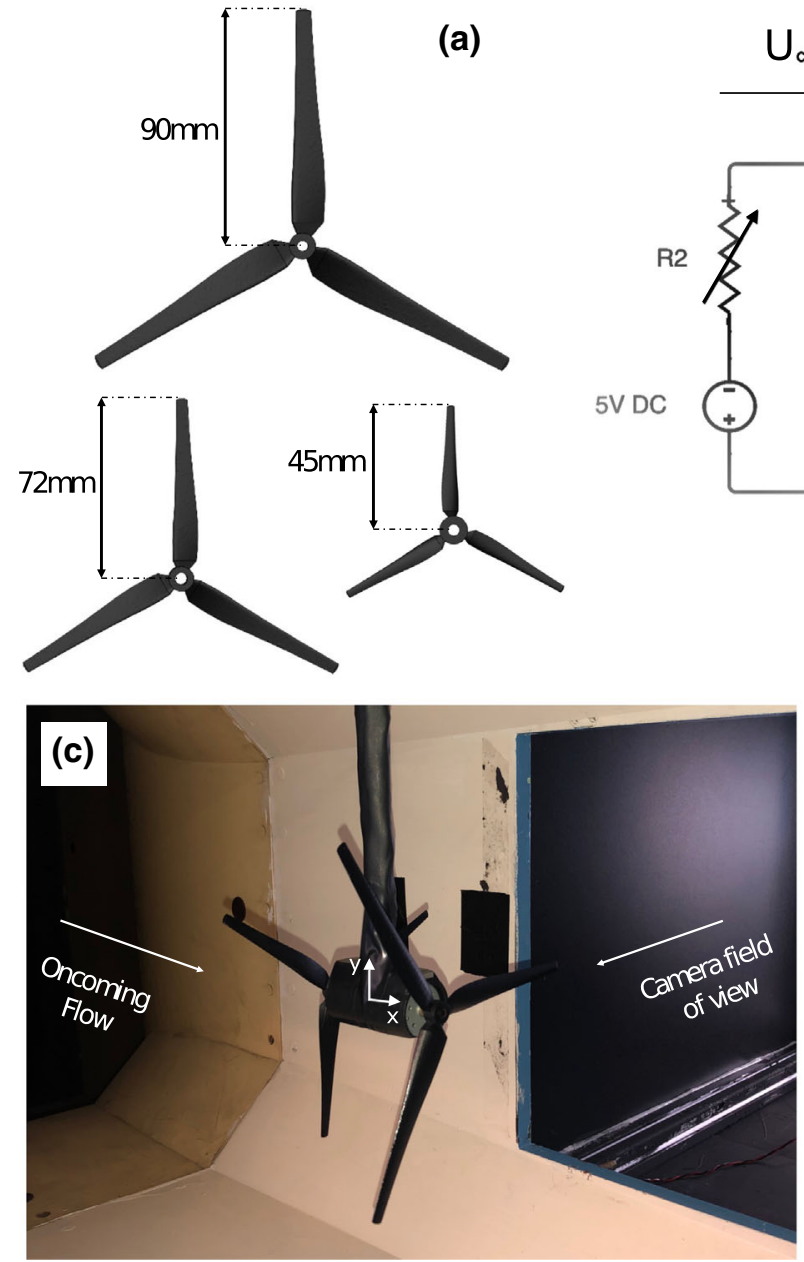

$U_{\infty}$

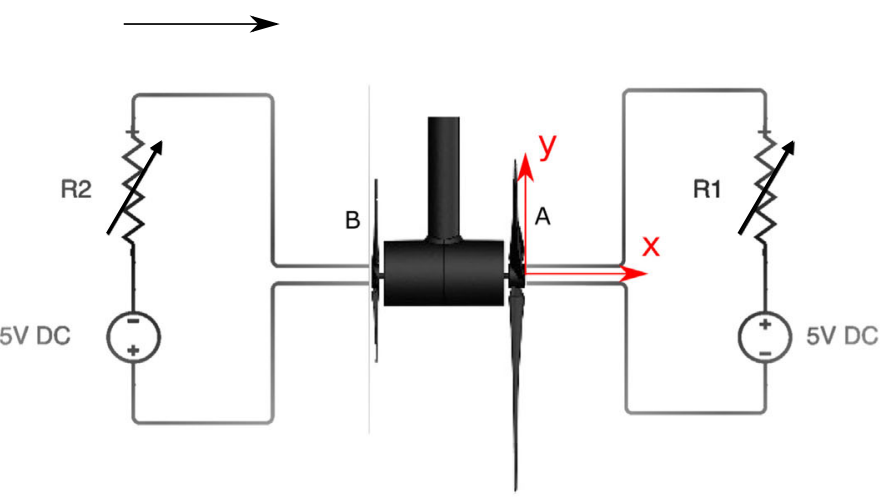

(b)

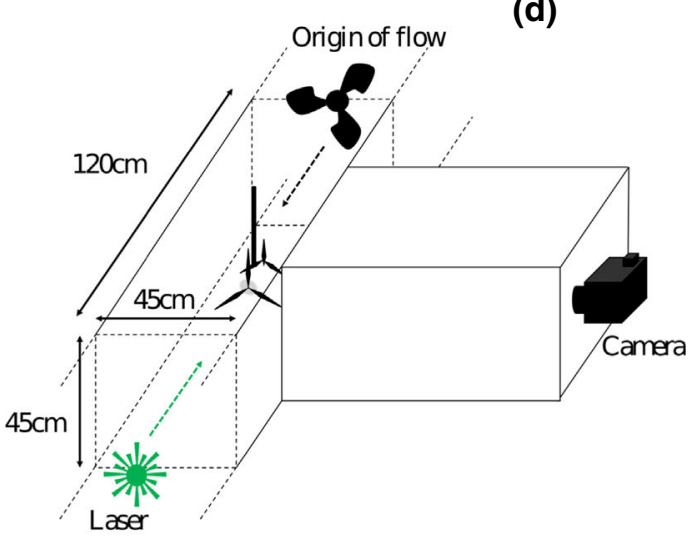

Fig. 1 a Rotor blades, b model circuit diagram, c, d experimental set-up photograph and schematic including wind tunnel working section dimensions 
the hub. As the superiority of the counter-rotating DRWT over the co-rotating DRWT and SRWT in power generation has been justified (Ozbay 2014; Wang et al. 2018 among others), only the counter-rotating condition is investigated in this study. Hereafter, DRWT refers to the counter-rotating configuration for short. The current SRWT configuration is different from a conventional one where the main rotor is installed on the windward side of the hub. The current SRWT configuration is to ensure a direct comparison to the two DRWT configurations. The conventional SRWT and DRWT configuration with the auxiliary rotor of the same size as the main rotor has been well-studied. Therefore, they are not repeated here.

In the context of this investigation, it is desirable that the results are independent of the Reynolds number (Re). Most wind tunnel experiments were conducted at lower Re than real conditions not surprisingly. Existing research into the Re dependence of turbulence statistics in the wake of turbines states that mean velocity and turbulence intensity, both of which are to be studied here, become independent of Re for $\operatorname{Re} \gtrsim 9.3 \times 10^{4}$ (Chamorro et al. 2012). Here, Re is defined as

$$
\operatorname{Re}=\frac{U_{\infty} D}{v}
$$

where $U_{\infty}$ is the free stream wind velocity at hub centre height, set to be $8 \mathrm{~ms}^{-1} ; D$ is the characteristic length scale of the flow, taken as the diameter of the main rotor and $v$ is the kinematic viscosity for air at $20{ }^{\circ} \mathrm{C}$. This gives $\mathrm{Re} \approx 9.5 \times 10^{4}$, satisfying the Re independence criterion.

The tip speed ratio $\Lambda$ is an important factor of wind turbine design (Yurdusev et al. 2006) which quantifies the power generation capability and consequent efficiency of a turbine. It is defined as the ratio between the blade tip speed and the free stream velocity, viz $\Lambda=\Omega L / U_{\infty}$, where $\Omega$ is the turbine rotational speed and $L$ is the length of the blade. According to Ragheb and Ragheb (2011), the optimal $\Lambda$ was found to be $\Lambda_{\text {opt }}=4 \pi / m$, where $m=3$ is the number of blades in this study.

A turbine with $\Lambda$ that is too low fails to capture energy from the wind. Alternatively, a rotor having too large $\Lambda$ acts as more of a barrier effect to the incoming air. It is shown by Siddiqui et al. (2017) that $\Lambda$ greatly impacts properties of the wake, namely velocity, vorticity and flow streamline trend, rendering it a parameter that must remain constant throughout the experimentation. However, $\Omega$ required for $\Lambda_{\text {opt }}$ from the $50 \%$ auxiliary rotor was too high to be feasible, and for this reason, a slightly lower value of $\Lambda=3.46$ was chosen and was set to be the same for all the rotors. At this value, the theoretical power coefficient for a turbine with three blades of NACA 4415 profile type, defined as the ratio of electrical power output to wind power input, is found to be 0.4196 (Yurdusev et al. 2006) well within the average range of 0.2-0.45.

A MFA como drill motor and a low inertia solar motor were used to control the rotor rotational speed. Each of them was connected to a rotor using a push-fit fastener and powered by a $5 \mathrm{~V}$ source. The connecting wires were wrapped around the turbine tower, properly secured so as to not alter its aerodynamic properties, and fed out of the working section. The rotors were powered to rotate in the direction they would naturally do in a free stream, as dictated by their blade profiles. As shown in Fig. 1b, adjustable resistors were used in order to tune the rotational velocity until the desired value was reached, measured using a strobe light. The accuracy provided by the strobe saw a change in tip speed ratio of maximum $\pm 0.5 \%$, deeming the set-up suitably reliable. Table 1 details the working conditions of the rotors, where $B$ is the auxiliary rotor upstream of the main rotor $A$.

\subsection{PIV measurements}

Two-dimensional particle image velocimetry (PIV) measurements were performed to investigate the near wake flow structure. The flow was seeded with oil droplets of diameter $\approx 1 \mu \mathrm{m}$ produced by an atomiser; they are small enough to follow the motion of the flow, large enough for PIV cross-correlation at the set field of view (FOV). Particles are distributed homogeneously in the FOV plane, illuminated by a $120 \mathrm{~mJ}$ per pulse $15 \mathrm{~Hz}$ double-headed Nd:YAG laser, which fired from far downstream of the flow as shown in Fig. 1c.

Table 1 Table of the working conditions for each configuration with reference to Fig. $1 \mathrm{~b}$

\begin{tabular}{llll}
\hline Configuration & Rotor $A \varnothing(\mathrm{mm})$ & Rotor $B \varnothing(\mathrm{mm})$ & $\Omega(\mathrm{RPM})$ \\
\hline SRWT & 180 & NA & 2936 \\
DRWT(L) & 180 & 144 & 3671 \\
DRWT(S) & 180 & 90 & 5866 \\
\hline
\end{tabular}

For the two DRWT configurations, only the $\Omega$ of the auxiliary rotor is listed, as the $\Omega$ for the main rotor is the same as that in the SRWT configuration. $L$ and $S$ denote large and small auxiliary rotor, respectively 
The laser sheet was set to $\approx 3 \mathrm{~mm}$ thick to account for the out-of-plane velocity component due to blade rotation. The PIV $\Delta t$ was set to $30 \mu$ s for FOV size of about $190 \mathrm{~mm} \times 240 \mathrm{~mm}$ in the $x-y$ plane, where $x$ is the streamwise direction and $y$ is along the rotor radius (vertical) direction. The origin was set at the leeward centre of the main rotor; see Fig. 1b. The FOV was offset in the $y$ direction to be optimised for the part of the wake away from the tower. A low speed SensiCam camera was used as the imaging tool, sampling at a rate of four image pairs per second and faced normal to the FOV in an enclosure. Figure 1c, d shows the image and the schematic of the set-up, respectively.

In total, 1000 pairs of images were taken from each of the configurations defined in Table 1 . The particle images were processed by LaVision ${ }^{\circledR D}$ Davis 7.0 , with $32 \times 32$ pixel interrogation window and $50 \%$ overlap. This gives the spatial resolution $\approx 3 \mathrm{~mm}$ by vector spacing. The instantaneous velocity in the $x$ and $y$ direction, respectively, is written as $(u, v)=\left(U+u^{\prime}, V+v^{\prime}\right)$, where $(U, V)$ is the ensemble averaged (time mean) velocity and $\left(u^{\prime}, v^{\prime}\right)$ is the fluctuating component.

\section{Results and discussion}

\subsection{Mean wake}

Figure 2 illustrates the mean streamwise velocity $U$ distribution over the region $-0.4<x / D<0.9$. Similar to most of the wind turbine wake studies, we focus on the part of the wake away from the tower. Note that in our study, the tower was installed upside down, due to physical constraints of the facility (Fig. 1c).

As expected, significant velocity deficit can be seen in the wakes behind all three configurations, indicating a large amount of the incoming flow's kinetic energy being consumed. The DRWT(L) featuring the larger auxiliary rotor shows the greatest velocity deficit, closely followed by the DRWT(S), both have greater deficit than SRWT at the end of the FOV. This is confirmed by the $U$ distribution along the radial direction, as it is shown in Fig. 3a. It shows that the free stream velocity $U_{\infty}$ is fully recovered at $|y / D|=$ 0.6 by $x=0.9 D$. Figure 2 shows that the velocity deficit area is roughly cone shaped, gradually expands starting at the main rotor tips. The slight overshoot of $U\left(>U_{\infty}\right)$ at $y / D=-0.6 \mathrm{in} \mathrm{Fig.} \mathrm{3a} \mathrm{is} \mathrm{because} \mathrm{of} \mathrm{the}$ induced velocity caused by the three winding helical vortex cores originating from the main rotor blades, which will be investigated later. It shows that the width of the wake for the three configurations are very similar, with the wake of DRWT(L) marginally wider. This is consistent with the findings of Ozbay (2014) who also found the wake width behind an SRWT and DRWT to be almost identical and consequently dependent on the size of the main rotor, which has also been kept constant there. This establishes that it is not the wake shape that changes with the addition of an auxiliary rotor, but the characteristics within it, further supported visually by Fig. 2 .

The fact that the obvious quicker velocity recovery is seen in the SWRT for $-0.5 \lesssim y / D \lesssim-0.2$ (Fig. 3a) confirms that the auxiliary rotors of the DRWTs capture a large proportion of the kinetic energy of the flow at the main rotor root region, otherwise missed by the SRWT. This is consistent with existing knowledge that DRWTs are able to yield a higher power output than conventional horizontal-axis SRWTs (Ozbay 2014; Herzog et al. 2010). Over the same region, Fig. 2 shows that DRWT(S) has the highest
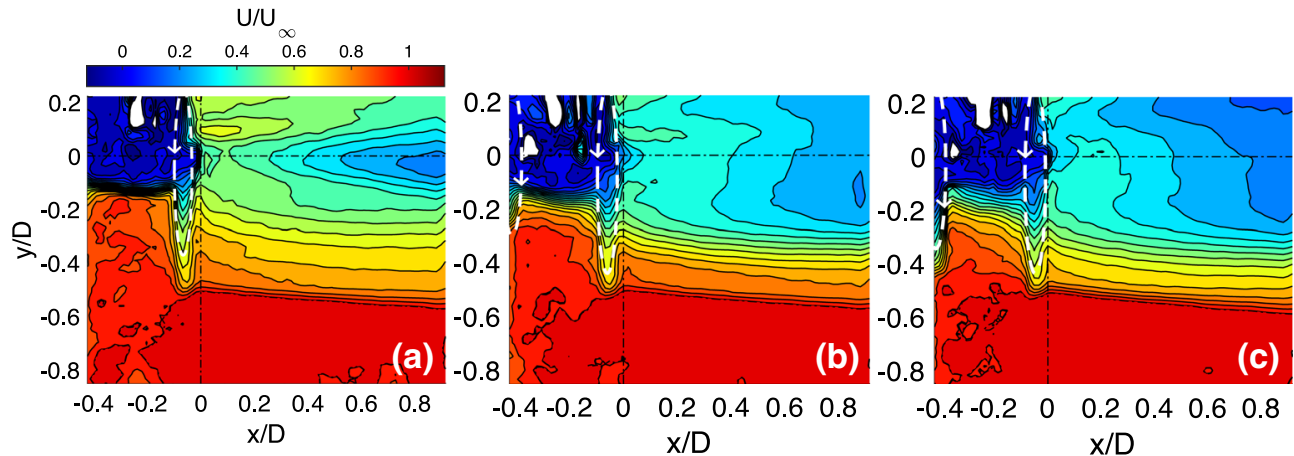

Fig. 2 Contour of the mean streamwise velocity. a SRWT, b DRWT(S), c DRWT(L). The position of the rotors are labelled with the white arrows indicating the rotational direction (viewing from downstream for the main rotor and from upstream for the auxiliary rotors, so they counter-rotate) 

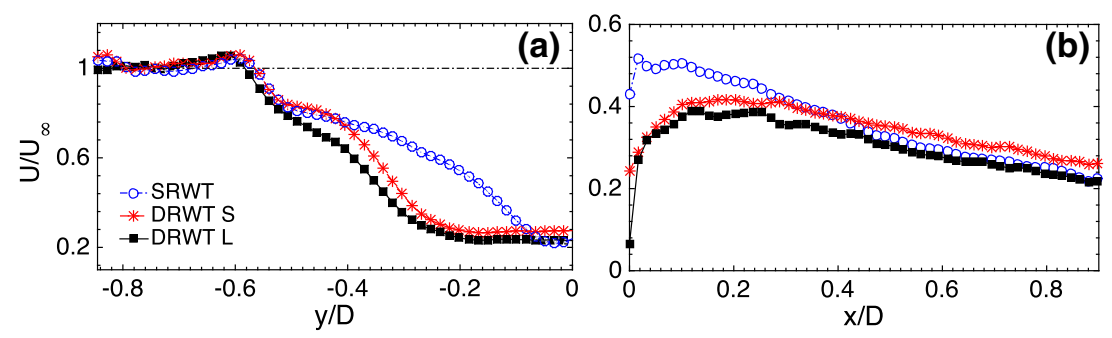

Fig. 3 a Dependence of axial velocity on the radial distance from the hub centre at $x=0.9 D, \mathbf{b}$ dependence of the axial velocity on the axial distance at hub centre height

density of contour lines followed closely by DRWT(L). This can also be inferred by $\partial U / \partial y$ derivable from Fig. 3a. This suggests that the velocity gradient in the radial direction, and therefore shear strength, is larger for the DRWTs when compared to the SRWT. Ozbay (2014) demonstrated that the presence of high shear is prone to flow instability and hence promotive of turbulent mixing. This turbulent mixing is desired to breakdown the cone-shaped shear layer, caused by tip vortices as shown in Sect. 1, in order to accelerate the process of wake recovery.

Figure $3 \mathrm{~b}$ shows the $U$ deficit with axial distance at hub height. The profiles differ significantly within the region $0<x / D<0.3$, wherein the auxiliary rotors of the DRWTs resulting in a larger velocity deficit in the immediate near wake. Beyond this distance, $U$ behaviours are very similar among the three. By the end of the measurement range, $U$ at hub height continues dropping, but it can be expected that it will eventually recover to $U_{\infty}$ as the wake dissipated. Whether or not the $U$ distributions of the three remain similar further downstream requires further investigation.

Figure $3 \mathrm{~b}$ also shows that $U$ of the DRWT(L) is consistently lower than the other two configurations, suggesting the former is slightly superior at extracting energy from the wind. This agrees with the findings of Jung et al. (2005) who stated that using a secondary rotor $\approx 50 \%$ the size of the main rotor sees the best performance in the context of power output, yielding the highest power coefficient.

\subsection{Phase-averaged wake}

As the rotation rate of the rotors is fixed, it is expected that the wake behind all the configurations manifests a periodic feature, at a frequency of $m \Omega /(2 \pi)$. Since the samples were acquired at a fixed low frequency in a statistically independent way, without phase locking by an external phase indicator, the phase of the wake is resolved using the snapshot-based proper orthogonal decomposition (POD) (Berkooz et al. 1993). POD is a suitable but not unique tool to extract coherent flow structures from both turbulent velocity data, e.g. Wang et al. (2020), and passive scalar data, e.g. He and Liu (2017). [Other techniques, e.g. wavelet transform (Fijimoto and Rinoshika 2017), might also be suitable for the similar purposes under special circumstances.]

Briefly speaking, for a set of $N$ snapshots of the fluctuation components, $\left(u^{\prime}, v^{\prime}\right)$, an auto-covariance matrix $M$ is constructed where solving the standard eigenvalue problem produces $N$ eigenvalues $\lambda_{i}$, and $N$ eigenvectors $A_{i}$.

$$
M A_{i}=\left(\hat{U}^{T} \hat{U}\right) A_{i}=\lambda_{i} A_{i}
$$

where $\hat{U}=\left[u_{1}^{\prime} \ldots u_{N}^{\prime}, v_{1}^{\prime} \ldots v_{N}^{\prime}\right]$, combining both velocity components. The associated POD mode, $\Phi_{i}$ can be calculated as:

$$
\Phi_{i}=\frac{\sum_{n=1}^{N} A_{i, n}\left(u_{n}^{\prime}, v_{n}^{\prime}\right)}{\left\|\sum_{n=1}^{N} A_{i, n}\left(u_{n}^{\prime}, v_{n}^{\prime}\right)\right\|}, i=1,2, \ldots N .
$$

The eigenvalue $\lambda_{i}$ reflects the contribution of mode $\Phi_{i}$ to the total fluctuating energy of the flow. The instantaneous velocity field can then be represented as a sum of orthogonal modal contributions as 


$$
(u, v)=(U, V)+\sum_{i=1}^{N} a_{i} \Phi_{i}
$$

where $a_{i}$ is the coefficient that is obtained by projecting the instantaneous velocity fields on the POD basis. That is

$$
a_{i}=\left[\begin{array}{lll}
\Phi_{1} & \Phi_{2} \ldots & \Phi_{N}
\end{array}\right]^{T}\left(u_{i}^{\prime}, v_{i}^{\prime}\right) .
$$

The ranking of the modal energy contribution, viz. $\lambda_{i}$ percentage, is given in Fig. 4a. It shows that $\lambda_{1}$ and $\lambda_{2}$, corresponding to $\Phi_{1}$ and $\Phi_{2}$, in total contribute 3.2\%,3.3\% and 3.5\% of the energy for the SRWT, DRWT(S) and DRWT(L) configurations, respectively, which are fairly similarly small. Higher modes contribute less than $1.5 \%$ each. This means that the wake behind all the three turbine configurations is very turbulent, and the energy contained in the coherent structures is relatively small. Contribution of $\lambda_{1}-\lambda_{4}$ of the two DRWTs are similar, more than that of SRWT. From $\lambda_{5}$ onwards, all the configurations become very similar in $\lambda_{i}$. SRWT gains a small fraction back at higher mode $i \gtrsim 25$, which are unimportant due to incoherency.

Figure $4 \mathrm{~b}$ illustrates the projection of the (normalised) $a_{1}$ and $a_{2}$ on to the polar coordinates, with the associated mode $\Phi_{1}$ and $\Phi_{2}$ presented in (c), in terms of vorticity derived from the modal velocity. It is evident from (c) that the first two modes reflect a periodic vortex shedding pattern, and this is confirmed by the rather homogeneous angular distribution in (b). This suggests that $a_{1}=\sqrt{2 \lambda_{1}} \sin (\phi)$ and $a_{2}=$ $\sqrt{2 \lambda_{2}} \cos (\phi)$ with $\phi$ representing the vortex shedding phase angle (Oudheusden et al. 2005). Phase averaging can be done by defining a sample bin size, in this study $\pm 10^{\circ}$, to ensure a sample size of $55 \pm 5$ in each bin (phase). An example of a bin centred at an arbitrary phase is shown in (b). At this phase, the averaged vorticity field $(\omega)$, using the raw instantaneous velocity $(u, v)$, is shown in (d) and the contour of the swirling strength $\lambda_{c i}$ is shown in (e). $\lambda_{c i}$ is the imaginary part of the complex eigenvalue of the (phaseaveraged) velocity gradient tensor, which provides a measure of the swirl strength to allow shear layer to be excluded from detections (Zhou et al. 1999).

The coherent vortices shed from the main rotor tips are clearly shown in Fig. $4 \mathrm{~d}$ after phase averaging. Also seen is the area of vorticity originates from the small auxiliary rotor. These vorticity, in the form of shear layer, fails to form any coherent vortex packets due to the interference of the main rotor downstream. At the same tip speed ratio $\Lambda$, the auxiliary rotor and the main rotor rotate at different rates, having different vortex shedding frequency captured in FOV. It is confirmed in (e) that no strong swirl is observed in this area, while clear swirling vortex cores can be seen downstream of the main rotor. POD analysis of the subregion excluding the main rotor vortices also do not show evidence of periodic shedding from the auxiliary rotor; figure not shown.

Figure 5 shows three successive wake phases from $\phi=\pi / 4$ behind all three configurations. As the phase angle increases, the tip vortices shed from one main rotor blade can be seen to align sequentially with the vortices shed from the other two blades of the same rotor. The distance between the neighbouring vortices is
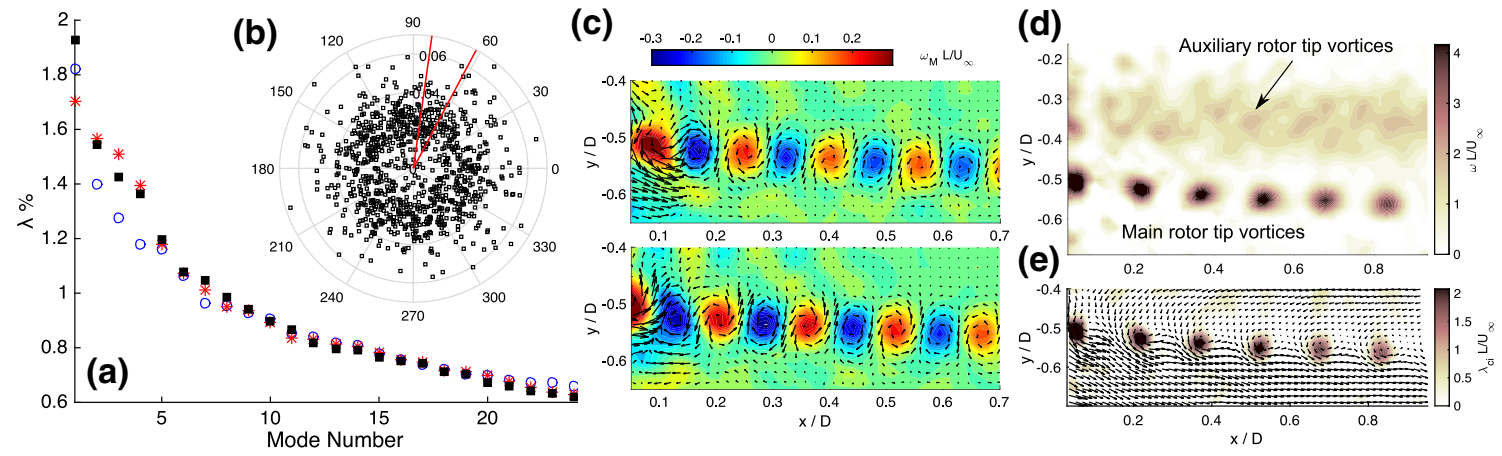

Fig. 4 POD analysis of DRWT(S) dataset. a Percentage of the POD mode energy to the total energy. Mode zero, viz. energy of the mean flow fields is excluded. Legends follow Fig. 3, b projection of the normalised coefficients of the first two modes $a_{1} / \sqrt{2 \lambda_{1}}$ and $a_{2} / \sqrt{2 \lambda_{2}}$ from each snapshot on to the polar coordinates. The red lines mark the $\pm 10^{\circ}$ bin size for phase averaging, $\mathbf{c}$ the vorticity $\omega_{M}$ contours of the first two modes $\Phi_{1}$ and $\Phi_{2}$, overlaid with the corresponding modal velocity vectors, $\mathbf{d}$ phase-averaged vorticity contour from the snapshots falling inside the bin in (b), e the corresponding phase-averaged swirling strength $\lambda_{c i}$ 
found to be fairly constant among the three configurations and over the entire FOV, which is about $0.15 \mathrm{D}$. Since the vortices are convected downstream by the local velocity and the rotation rate of the main rotor remains constant, this suggests that the auxiliary rotor does not impact on the local mean velocity in the wake, in agreement with Fig. 2.

The trace of the turbine root vortices is also observable in the wake behind SRWT, but not in a coherent pattern in-phase with the tip vortices. This might be because of the particular blade shape used inhibiting coherent vortex shedding in the root part. In comparison, the influence of the smaller auxiliary rotor is clearly seen in the wake of DRWT(S), where stronger vorticity is seen as highlighted by the blue box. This shear layer is also reflected in the $U$ profile at the end the FOV in Fig. 2a, where a 'step' is seen for $0.4 \lesssim|y / D| \lesssim 0.5$. This shear layer has the same sense of direction as the main rotor tip vortices in the $x-$ $y$ plane, but should have an opposite swirl direction as the main rotor helical wake due to the counterrotating auxiliary rotor. This could be beneficial as it counteracts the main rotor tip wake swirl.

The strength of this shear layer is appreciably lower in DRWT(L). This is because the vortices shed by the larger auxiliary rotor are entrained into the main rotor vortices, attributed to their closer radial distance. This is also the reason for the stronger vortices (both size and $\omega$ magnitude) behind DRWT(L). The vortex interaction also tends to distort the shape of the vortices for $x>0.7 D$.

The negative $\omega$ is negligible hence not included in Fig. 5. Note that although the auxiliary rotor rotates in the opposite direction as the main rotor, because of its mirrored blade geometry, the two rotors shed vortices in the same sense in $x-y$ plane.

It is so far clear that the main rotor tip vortices still play a dominant role, acting as a barrier preventing wake re-energisation. Its evolution is further analysed next. The vortex centre trajectory is shown in Fig. 6a. The vortex centre is found by the $\lambda_{c i}$ weighted centroid; see Fig. 4e. The trajectory behind all three configurations are very similar, with a very small rate of expansion, weakly increasing from SRWT, DRWT(S) to DRWT(L), under the influence of the auxiliary rotor vortices.

Figure $6 \mathrm{~b}$ displays the decay of circulation $\Gamma$ of the tip vortex packets, where $\Gamma=\int_{S} \omega \mathrm{d} s$ for $S$ denoting the vortex packet area based on a universal threshold $\omega L / U_{\infty}=0.4$, about $6 \%$ of the peak vorticity value in Fig. 5. The $\Gamma$ decay can be well-described by an exponential function $\Gamma=\Gamma_{0} \exp \left[-\alpha_{\Gamma}(x / D)\right]$. At the main

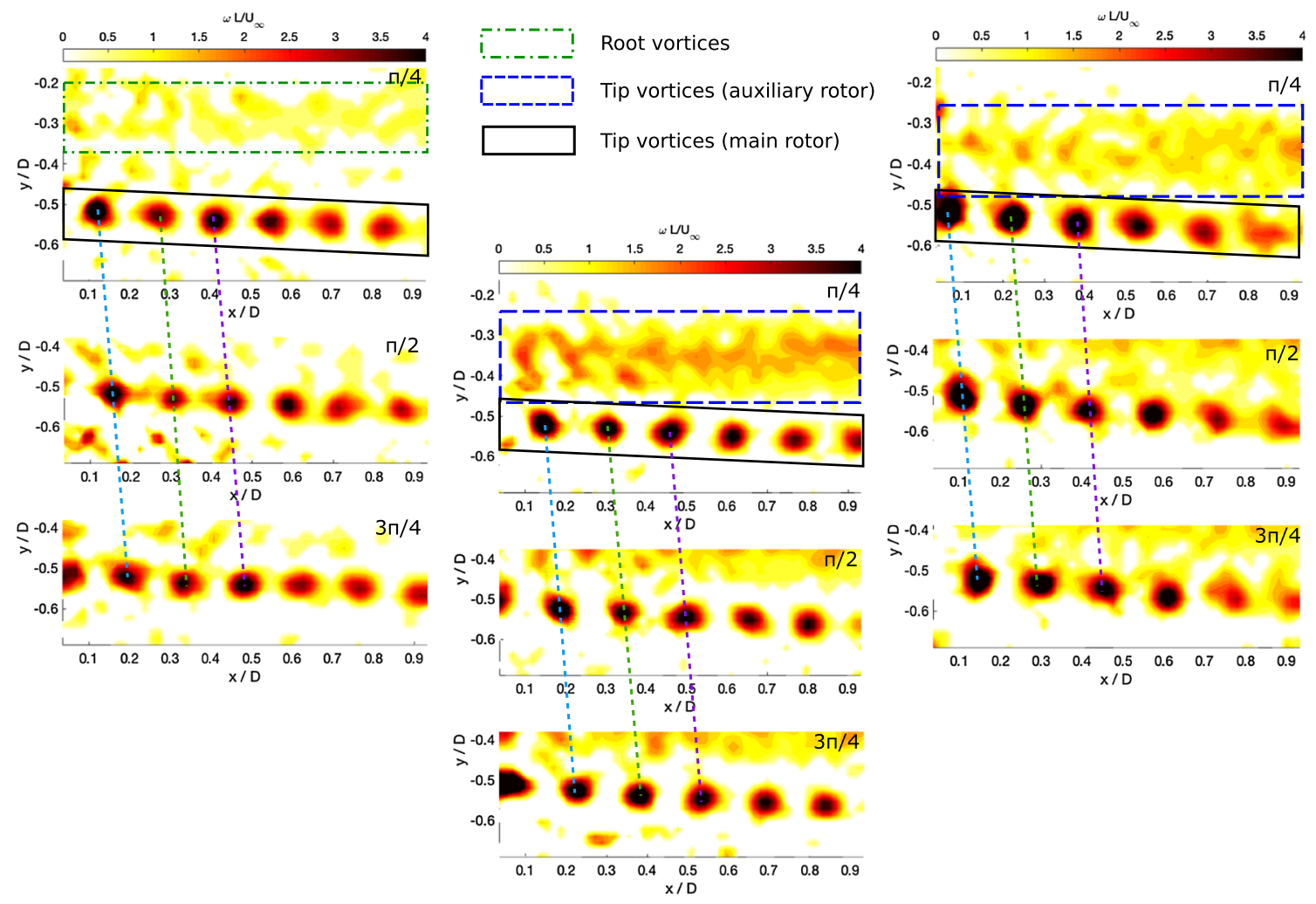

Fig. 5 Phase-averaged vorticity contour for three successive phase angles behind all three turbine configurations. From left to right: SRWT, DRWT(S), DRWT(L) 


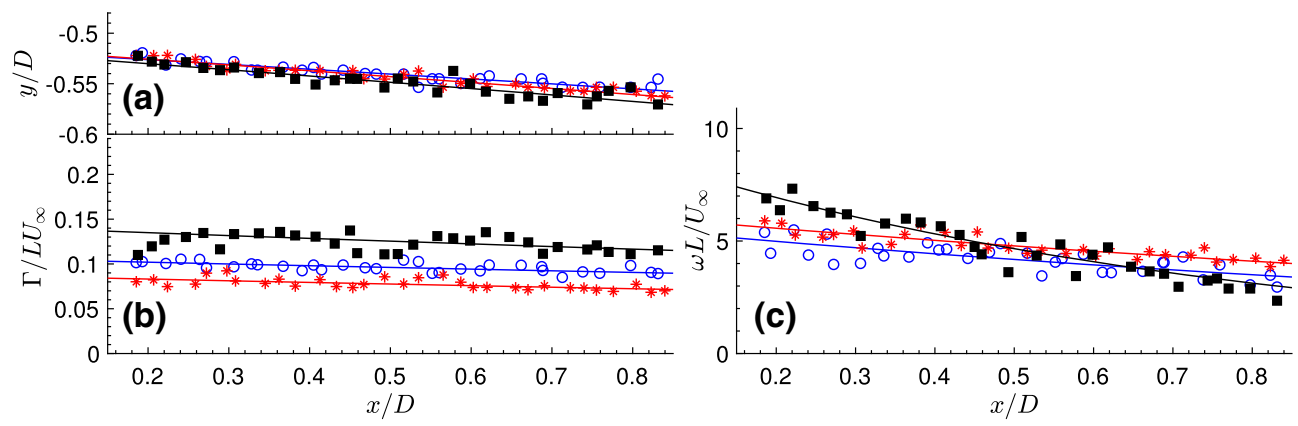

Fig. 6 a The trajectory of the main tip vortex centre, $\mathbf{b}$ dependence of the main rotor tip vortex circulation $\Gamma$ on the streamwise distance. Legends follow Fig. 3, c dependence of the vorticity at vortex centroids on the streamwise distance. The solid lines are the least squares exponential fit to the data points

rotor tips $x=0, \Gamma_{0} / L U_{\infty}=0.11,0.087$ and 0.141 for SRWT, DRWT(S) and DRWT(L), respectively. In consistency with Fig. 5, DRWT(L) have the strongest vortices in the wake, due to the auxiliary rotor vortices entrained and also the weaker vorticity connected with the auxiliary vortices shear region. Interestingly, DRWT(S) has the vortices of the lowest $\Gamma$, even lower than SRWT. Close examination of Fig. 5 suggests that the influence of the smaller auxiliary rotor is to take the background vorticity near the main rotor vortices away from them and deliver that to the root vortices area.

The $\Gamma$ decay rates are found to be similar among the three, with $\alpha_{\Gamma}=0.20,0.23$ and 0.24 , respectively. In particular for the two DRWTs, their decay rates are nearly identical. This suggests that the size of the auxiliary rotors does not have a large impact on the tip vortices decay rate. However, compared to SRWT, incorporation of the auxiliary rotors does increase it, very weakly, due to the vortex interaction.

Similarly, the peak vorticity at the vortex centroids also displays an exponential decay described by $\omega_{p}=\omega_{0} \exp \left[-\alpha_{\omega}(x / D)\right]$; see Fig. 6c. The decay rate $\alpha_{\omega}=0.60,0.51$ and 1.33 for SRWT, DRWT(S) and DRWT(L), respectively. It is clear that DRWT(L) sees the most rapid $\omega_{p}$ decay, while that for SRWT and DRWT(S) is similar. If the $\omega$ profile of the vortices are assumed to be close to Gaussian, it is possible to deduce the $x$ dependence of the characteristic vortex size $r$, combining the $\Gamma$ and $\omega_{p}$ behaviour. That is $r \sim \exp \left(\alpha_{r} x\right)$, where $\alpha_{r}=\left(\alpha_{\omega}-\alpha_{\Gamma}\right) / 2=0.2,0.14$ and 0.55 . This means that $r$ gradually increases due to vorticity diffusion, and this rate is the fastest for DRWT(L). At $x=0$, extrapolation of the exponential relations find $\omega_{0} L / U_{\infty}=5.6,6.1$ and 9.0 for the three configurations, respectively.

\subsection{Turbulence kinetic energy}

Finally, we take a look into the fluctuating velocities. Without knowing the out-of-plane velocity component $w$, TKE in this study is defined as

$$
\mathrm{TKE}=\frac{1}{2}\left(\overline{u^{\prime} u^{\prime}}+\overline{v^{\prime} v^{\prime}}\right),
$$

where $\overline{u^{\prime} u^{\prime}}$ and $\overline{v^{\prime} v^{\prime}}$ are the normal stress in the $x$ and $y$ directions, respectively. Figure 7 depicts TKE contour for all three turbine configurations. Consistent with the finding that the wake width is mainly dependent on the vortex core trajectory, and is therefore very similar behind the three turbines, the TKE distribution patterns are also similar. Very close to the main rotor surface, the TKE intensity behind SRWT appears higher than the two DRWTs, where part of the free stream wind energy is absorbed by the auxiliary rotors. In DRWT(S), higher TKE intensity can vaguely be seen just above and below the vortex core trajectory, while in DRWT(L) higher TKE can only be seen below. This is in line with the visualisation shown in Fig. 5, where auxiliary rotor vortices are entrained to the main rotor vortices behind DRWT(L).

Figure 8 demonstrates the change of the fluctuating velocity root mean square, $u(\mathrm{rms})$, along the vortex centroid trajectory, where $u(\mathrm{rms})=\sqrt{\mathrm{TKE}}$. Up to the end of the FOV, the magnitude and the decay rate of $u(\mathrm{rms})$ are found to be similar among all the three configurations. For $x>0.5 D, u(\mathrm{rms})$ is the highest for DRWT(L) and lowest for DRWT(S). This is in agreement with the evolution of the $\Gamma$ magnitude shown in Fig. $6 \mathrm{~b}$. The fluctuating velocity $\left(u^{\prime}, v^{\prime}\right)$ is obtained from subtracting the time mean from the instantaneous velocities, and hence consists of both coherent mean (for periodic flows) and random turbulence. High random turbulence intensity contributes to the dissipation of helical wake and consequent wake re-energisation deeming it, at appropriate regions, a desirable quantity for the application. The phase-averaged 

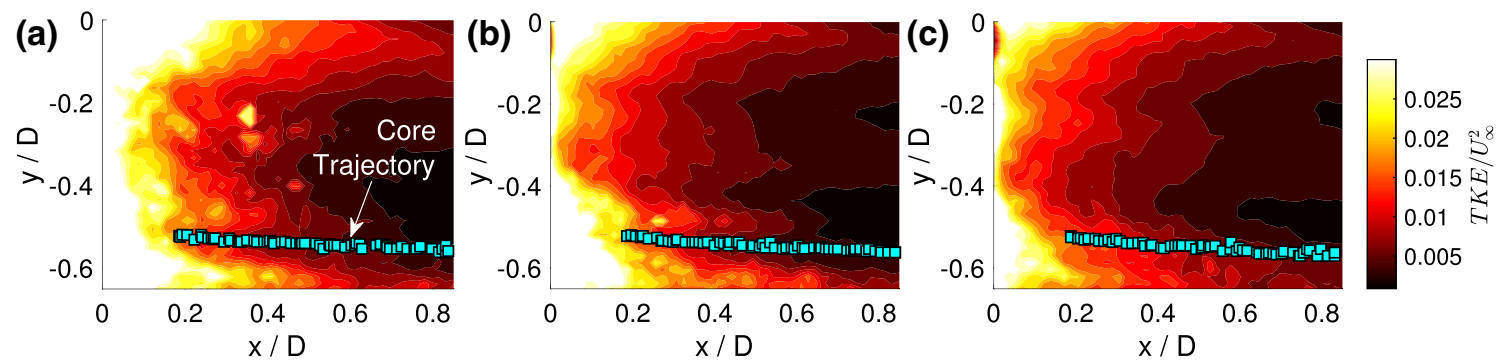

Fig. 7 Contour of TKE overlaid with the vortex core trajectory taken from Fig. 6a. a SRWT, b DRWT(S) and c DRWT(L)

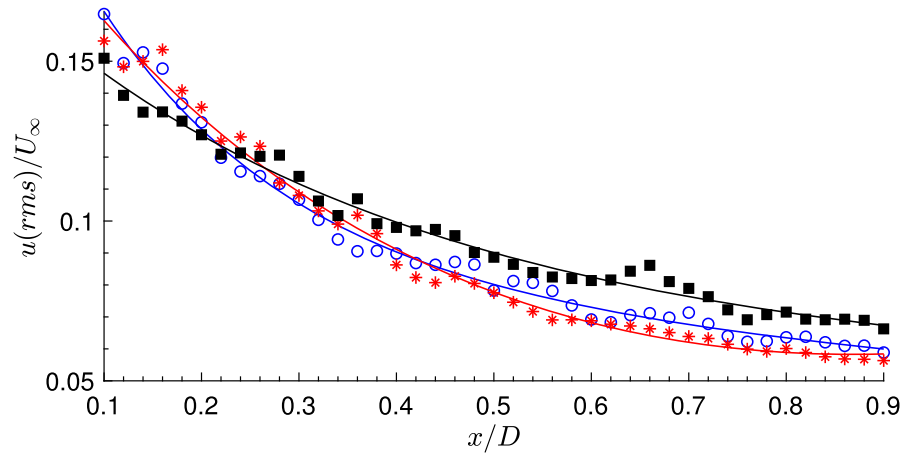

Fig. 8 Fluctuating velocity rms along the vortex core trajectory. Solid lines are the least squares fits. Legends follow Fig. 3

vortices discussed in Sect. 3.2 are coherent mean which contribute significantly to the fluctuating velocities. The $u(\mathrm{rms})$ intensity around the vortex cores is thus a manifestation of the circulation $\Gamma$ of the vortex packets. The similar $u(\mathrm{rms})$ decay rates for $x>0.5 D$ cannot be fitted with a simple exponential function. However, they are also in consistence with the decay rate of $\Gamma$ in Fig. 6 a.

\section{Conclusion}

The near wake velocity field behind three turbine configurations was experimentally studied in order to evaluate the impact of an additional counter-rotating auxiliary rotor on the conventional single-rotor wind turbine wake. The two auxiliary rotors were of $80 \%$ and $50 \%$ scale of the main rotor, installed upwind of the main rotor, aiming to capture the energy loss in the root part of the latter. All the rotors were tested at a constant tip speed ratio 3.46. We focused on the wake region within one main rotor diameter behind the turbines. Characteristics of the mean velocity field, phase-averaged vortices and TKE were studied using 2DPIV. The following conclusions may be established:

- Incorporating auxiliary rotors induces greater mean velocity deficit, with DWRT(L) marginally larger than DRWT(S), meaning that more wind energy was utilised with an auxiliary rotor.

- The size of the auxiliary rotor does not impact very much on the width of the wake, which primarily is determined by the trajectory of the vortices shed by the main rotor installed at the downwind side, in agreement with Ozbay (2014). The vortices shed by the 50\% scale auxiliary rotor leaves a shear layer behind without coherent structures surviving the main rotor interference. Those shed at the $80 \%$ scale rotor are entrained into the main rotor vortices.

- The DRWT(L) has the strongest main rotor tip vortices because of the entrainment of the auxiliary rotor vortices. Although it also experiences the most rapid decay of vorticity strength at the vortex core centroids, very obviously, the decay rate of its vortex circulation is only marginally larger than the other two configurations. The decay of peak vorticity and vortex circulation is found to be exponential for all three configurations.

- In line with the circulation behaviour, DRWT(L) sees the slightly strongest TKE intensity at the vortex core trajectory, but the decay rate is similar to the other two configurations. 
- Overall, the two DRWTs do not see a significant difference in the near wake characteristics. DRWT(S) with 50\% scaled auxiliary rotor seems to work better owing to its weaker vortex circulation and TKE along the vortex trajectory. Although it results in a strongest shear layer behind the mid-span of the main rotor, this wake is believed to be beneficial because of its opposite swirl direction (counterrotating) which tends to counteract the main rotor wake. This is largely consistent with the findings of Jung et al. (2005) who found that an auxiliary rotor 40-50\% the size sees the best performance in the context of power output, when compared to rotors of other sizes.

Acknowledgements The authors would like to thank Mr. Lincoln Greatrick and Mr. Robbie Grout for their earlier contribution to the work; also the UK EPSRC (EP/P004377/1) for the financial support.

Open Access This article is licensed under a Creative Commons Attribution 4.0 International License, which permits use, sharing, adaptation, distribution and reproduction in any medium or format, as long as you give appropriate credit to the original author(s) and the source, provide a link to the Creative Commons licence, and indicate if changes were made. The images or other third party material in this article are included in the article's Creative Commons licence, unless indicated otherwise in a credit line to the material. If material is not included in the article's Creative Commons licence and your intended use is not permitted by statutory regulation or exceeds the permitted use, you will need to obtain permission directly from the copyright holder. To view a copy of this licence, visit http://creativecommons.org/licenses/by/4.0/.

\section{References}

Bartl J (2011) Wake measurements behind an array of two model wind turbines. Master's thesis, Norwegian University of Science and Technology, Department of Energy and Process Engineering

Berkooz G, Holmes P, Lumley L (1993) The proper orthogonal decomposition in the analysis of turbulent flows. Annu Rev Fluid Mech 25:539

Chamorro L, Arndt R, Sotiropoulos F (2012) Reynolds number dependence of turbulence statistics in the wake of wind turbines. Wind Energy 15(5):733

Fijimoto S, Rinoshika A (2017) Multi-scale analysis on wake structures of asymmetric cylinders with different aspect ratios. J Vis 20(3):519-533

Global Wind Energy Council. Global Wind Report 2017

Gomez-Elviraa R, Crespob A, Migoyab E, Manuelb F, Hernandezc J (2005) Anisotropy of turbulence in wind turbine wakes. J Wind Eng Ind Aerodyn 93:797

He C, Liu Y (2017) Proper orthogonal decomposition of time-resolved LIF visualisation: scalar mixing in a round jet. J Vis 20(4):789-815

Herzog R, Schaffarczyk A, Wacinski A, Zurcher O (2010) In: European wind energy conference EWEC

Jung S, No T, Ryu K (2005) Aerodynamic performance prediction of a $30 \mathrm{~kW}$ counter-rotating wind turbine system. Renew Energy 30(5):631

Lee S, Hogeon K, Soogab L, Son E (2012) Effects of design parameters on aerodynamic performance of a counter-rotating wind turbine. Renew Energy 42:140

Oudheusden B, Scarano F, Hinsberg N, Watt D (2005) Phase-resolved characterization of vortex shedding in the near wake of a square-section cylinder at incidence. Exp Fluids 39:86

Ozbay A (2014) An experimental investigation on wind turbine aeromechanics and wake interferences among multiple wind turbines. Master's thesis, Iowa State University

Ragheb M, Ragheb A (2011) Wind turbines theory-the Betz equation and optimal rotor tip speed ratio. In: Fundamental and advanced topics in wind power. Intech. https://doi.org/10.5772/21398

Rosenberg A, Selvaraj S, Sharma A (2014) A novel dual-rotor turbine for increased wind energy capture. J Phys Conf Ser 524:012078

Sharma A, Frere A (2010) Diagnosis of aerodynamic losses in the root region of a horizontal axis wind turbine. Technical report, General Electric Global Research Center Internal Report

Sherry M, Sheridan J, Jacono D (2013) Characterisation of a horizontal axis wind turbine tip and root vortices. Technical report 2. Springer, Berlin

Siddiqui M, Rasheed A, Kvamsdal T, Tabib M (2017) Influence of tip speed ratio on wake flow characteristics utilizing fully resolved CFD methodology. J Phys 854:012043

Wang Z, Ozbay A, Tian W, Hu H (2018) An experimental study on the aerodynamic performances and wake characteristics of an innovative dual-rotor wind turbine. Energy 15(147):94

Wang Q, Gan L, Xu S, Zhou Y (2020) Vortex evolution in the near wake behind polygonal cylinders. Exp Therm Fluid Sci 110:109940

Yurdusev M, Ata R, Cetin N (2006) Assessment of optimum tip speed ratio in wind turbines using artificial neural networks. Energy 31(12):2153

Zhou J, Adrian R, Balachandar S, Kendall T (1999) Mechanisms for generating coherent packets of hairpin vortices in channel flow. J Fluid Mech 387:353

Publisher's Note Springer Nature remains neutral with regard to jurisdictional claims in published maps and institutional affiliations. 